\title{
A STEREOPHOTOGRAMMETRIC METHOD FOR DETERMINING IN SITU CONTACT AREAS IN DIARTHRODIAL JOINTS, AND A COMPARISON WITH OTHER METHODS
}

\author{
G. A. ATEShian,* S. D. KWAK, ${ }^{*}$ L. J. Soslowsky† and V. C. Mow* \\ *Orthopaedic Research Laboratory, Departments of Mechanical Engineering and Orthopaedic Surgery, \\ Columbia University, New York, NY 10032, U.S.A. and TOrthopaedic Research Laboratories, \\ Departments of Surgery and Mechanical Engineering and Applied Mechanics, University of Michigan, \\ Ann Arbor, MI 48109-0486, U.S.A.
}

\begin{abstract}
Determination of contact areas in diarthrodial joints is necessary for understanding the state of stress within the articular cartilage layers and the supporting bony structures. The present study describes the use of a stereophotogrammetry (SPG) system [Huiskes et al., J. Biomechanics 18, 559-570 (1985) and Ateshian et al, $J$. Biomechanics 24, 761-776 (1991)] for determining contact areas in diarthrodial joints, using a surface proximity concept similar to the one used by Scherrer et al. [ASME J. biomech. Engng 101, 271-278(1979)]. This method consists of evaluating the proximity of the articular surfaces to determine joint contact areas using precise geometric models of the joint surfaces obtained from the SPG system, and precise kinematic data, also obtained from SPG. In this study, the SPG method for determining contact areas is compared to other commonly used methods such as dye staining, silicone rubber casting and Fuji film contact measurement techniques which have been often used and reported by other investigators. The bovine glenohumeral joint and the bovine lateral tibiofemoral articulation (without the meniscus) were used to represent congruent and incongruent joints, respectively. While all the methods yielded consistent contact patterns for the incongruent tibiofemoral articulations, the results for the congruent bovine glenohumeral joints showed that the SPG and Fuji film methods were in better agreement than those obtained from the dye staining and silicone rubber casting methods. The advantages of the new SPG method are that it can be used for intact joints, and used repeatedly and quickly thus making contact-area movement analyses possible [Soslowsky et al., J. orthop. Res. 10, 524-534 (1992)]. The results of this comparison study show that the SPG technique is a reliable and versatile method for determining contact areas in diarthrodial joints.
\end{abstract}

\section{INTRODUCTION}

Determination of contact areas in diarthrodial joints is a necessary step toward understanding the effects of loading on articular cartilage. It has been hypothesized that large contact stresses, or changes in stress magnitudes and stress gradients, may lead to degenerative changes in cartilage (Howell et al., 1992). Thus, knowledge of high and low weight bearing regions can provide a better understanding of the etiology and pathomechanics of osteoarthritis. Contact measurements can be used not only to quantify normal joint contact areas, but also the subtle and gross alterations in contact areas which result from altered joint mechanics such as that due to muscle imbalances, osteochondral injuries, and ligamentous laxity or injury (e.g. Ahmed and Burke, 1983a, b; Brown and Shaw, 1983, 1984; Brown et al., 1991; Huberti and Hayes, 1984, 1988).

Various methods have been used to determine contact areas in diarthrodial joints. Early studies have described a dye injection method used for the determination of contact areas in the hip joint (Black et al., 1981; Greenwald and O'Connor, 1971). A similar technique which consists of injecting silicone rubber into the joint has been used in the patellofemoral and tibiofemoral joints (Kurosawa et al., 1980; Seedhom and Tsubuku, 1977; Walker and Erkman, 1975). More recently, a silicone oil-carbon black powder

Received in final form 12 April 1993. suspension squeeze, the ' $3 S$ technique', has been introduced (Yao and Seedhom, 1991).

Other methods provide contact pressure measurements as well as contact area data. One of these consists of placing discrete miniature piezoresistive transducers at various locations over one of the articular surfaces of a joint to measure transient contact loads during load application (Brown and Shaw, 1983, 1984; Manouel et al., 1992). Another technique requires the application of a micro-indentation transducer over the tibial and patellar surfaces (Ahmed. 1983; Ahmed and Burke, 1983a,b). This method uses a thin, plastically deformable film and is conceptually similar to the use of Fuji film (Prescale or Pressensor, Fuji Film Co. Ltd, Japan). The Fuji pressure-sensitive film, which is also inserted in the joint space, provides various intensities of red patterns which are related to the applied surface pressure. Although this technique has been utilized by many investigators (e.g. Baratz et al., 1988; Fukubayashi and Kurosawa, 1980; Haut, 1989; Huberti and Hayes, 1984, 1988; Singerman et al., 1987; Tencer et al., 1988), a comparison study of contact areas on the elbow joint has found it to be unsatisfactory when compared to silicone casting (Stormont et al., 1985).

There have also been reports of in vivo data on contact pressures between acetabular cartilage and an instrumented metallic hip endoprosthesis (Hodge et al., 1989). Another in vivo method, introduced by Scherrer and Hillberry (1979) and Scherrer et al. (1979), consists of evaluating the proximity of the articular surfaces to estimate contact areas using experimentally derived geometric models of the joint. In their study, the kinematic data representing the relative motion of 
the articular surfaces of canine shoulder joints in vivo were obtained from an instrumented three-dimensional spatial linkage inserted percutaneously into the humerus and scapula. Following sacrifice, the articular surfaces were exposed by dissection, and plaster castings of the joint surfaces were made from a silicone rubber mold. These castings were then digitized using a high-accuracy three-dimensional digitizer and geometric models of the surfaces were created by surfacefitting the data with bicubic splines (Scherrer and Hillberry, 1979). Spatial transformations, derived from the kinematic linkage data, were subsequently operated upon the mathematical models of the surfaces. These transformations realign the articular surfaces into the relative positions that they assumed in vivo for various canine shoulder joint angles. Contact areas were subsequently estimated by numerically determining the points of closest proximity between the opposing surfaces (Scherrer, 1977; Scherrer et al., 1979).

In the present study, a stereophotogrammetric (SPG) contact method is introduced, which is conceptually similar to the technique developed by Scherrer and coworkers (Scherrer, 1977; Scherrer and Hillberry, 1979; Scherrer et al., 1979), but differs from that technique in several methodological aspects. (1) The kinematic data relating articular surfaces in three-dimensional space are derived from optical targets which are rigidly fixed to the bones of the joint. The spatial coordinates of these targets are measured with a SPG system described by Huiskes et al. (1985) and Ateshian et al. (1991), and the transformation matrices relating the various joint positions are calculated using a least-squares analysis. (2) The geometric models of the joint surfaces are also obtained from SPG. Thus, in this SPG contact method, the measurements of joint kinematics and joint surface topography are obtained with similar measurement accuracies. (3) New robust computational algorithms for surface proximity evaluations are presented, which can handle articular surfaces of complex topography. The various aspects of this new SPG contact method are described in detail below.

This joint contact area determination method is compared to selected measurement techniques described above, namely the dye staining, silicone rubber casting, and Fuji film techniques. These methods are applied to the determination of contact areas in bovine glenohumeral joints and lateral tibiofemoral articulations (without the meniscus). These two joints are characterized, respectively, by highly congruent contact and incongruent contact.

Thus, the objective of this study is to introduce the methodology of the SPG contact technique, and compare the performance of this technique to other contact methods often used in biomechanics of diarthrodial joints. The complex task of comparing contact area results from various techniques is addressed in this study, and a comparison method which makes use of SPG is presented.

\section{CONTACT MEASUREMENT METHODS}

\section{Stereophotogrammetric contact}

In this method, two sets of seven optical 'alignment' targets, which are mounted on a semi-circular Plexiglas frame, are rigidly fixed to each bone of the joint using $2.5 \mathrm{~mm}$ Steinmann pins and polymethylmethacrylate (PMMA). The orientation of these targets is important since they must be visible from two orthogonal view directions as described below (Fig. 1). The joint is mounted on a loading apparatus, and a ceramic calibration frame, fitted with 16 targets of known three-dimensional coordinates ("calibration' targets, $\pm 6 \mu \mathrm{m}$ ), is placed immediately in front of the joint, such that the two sets of optical alignment targets are visible through a $12.7 \times 12.7 \mathrm{~cm}$ opening in the calibration frame. At each joint load or joint position, a pair of large format $(20 \times 25 \mathrm{~cm})$ photographs (a stereogram) of the calibration frame and

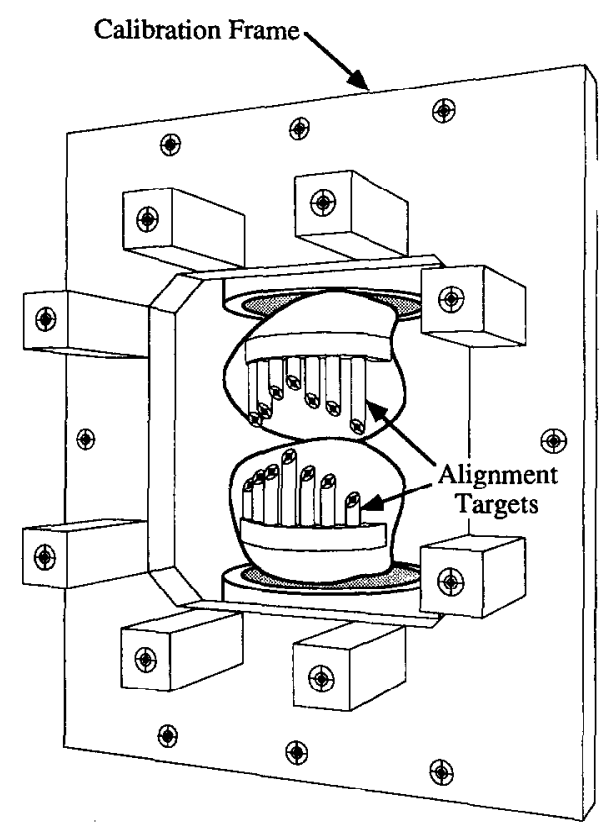

Fig. 1. A calibration frame is placed in front of the joint such that the alignment targets fixed to each bone are visible through the workspace of the frame.

alignment targets are obtained from two convergent directions, for subsequent SPG analysis (Soslowsky et al., 1992).

At the completion of the loading studies, the joint is dissected and the articular surfaces are exposed. The exposed articular surface of each bone is placed inside the workspace of the calibration frame, such that again the alignment targets for that bone are also visible relative to its cartilage surface. This can only be achieved if the placement of the alignment targets relative to the articular surfaces has been performed appropriately during specimen preparation, as mentioned above. The three-dimensional topography of the articular surface and the coordinates of the alignment targets are subsequently quantified with SPG, following a methodology described previously (Ateshian et al. 1991, 1992; Huiskes et al., 1985; Soslowsky et al., 1992). The same analysis is performed to reconstruct the three-dimensional coordinates of the alignment targets from the stereograms obtained in the loading studies. The uncertainty of these SPG measurements is $90 \mu \mathrm{m}$ at a $95 \%$ confidence level (Ateshian et al., 1991).

Geometric models of articular surfaces. Three-dimensional geometric models of the articular surfaces are obtained using parametric space surfaces. Each set of surface data is smoothed with a single fourth-order B-spline patch (Ateshian, 1993). The smoothed nodal data are fitted with a network of bicubic patches (Scherrer and Hillberry, 1979) forming a composite surface where first-order continuity $\left(C^{1}\right)$ is enforced at the boundaries of adjoining patches. Each bicubic patch is represented by $\mathbf{x}(u, w)$, where $\mathbf{x}$ is the position vector of any point on the patch at the parametric coordinates $(u, w)(0 \leqslant u \leqslant 1,0 \leqslant w \leqslant 1)$ (e.g. Ateshian, 1991). While bicubic patches wcre uscd in this study, any parametric surface equation of the form $\mathbf{x}(u, w)$ may be employed. The two surfaces of the joint are denoted by $S_{1}$ and $S_{2}$. The vectors $\mathbf{p}_{1, r}\left(r=1-n_{1}\right)$ and $\mathbf{p}_{2, s}\left(s=1-n_{2}\right)$ denote the threedimensional coordinates of the smoothed surface nodes on each surface, where $n_{1}$ and $n_{2}$ are the total number of nodes on each surface, respectively.

Surface realignment. The objective of this procedure is to place the geometric models of the articular surfaces into the positions that the actual surfaces assumed in the various joint 
loading configurations. This objective is accomplished utilizing the precise alignment target coordinates, both with respect to the loaded configurations and to the articular surface nodes. Let $t_{1, k}$ and $t_{2, k}(k=1-7)$ denote the alignment target coordinates when measured from the same stereogram as the corresponding surface nodes, and let $\mathbf{t}_{1, k}^{(1)}$ and $\mathbf{t}_{2 . k}^{(1)}$ correspond to the same targets as measured from the stereogram of some load configuration $(l)$. The spatial transformations which relate $t_{1, k}^{(1)}$ with $t_{1, k}$ and $t_{2, k}^{(l)}$ with $t_{2, k}$ are given by

$$
\mathbf{t}_{1, k}^{(l)}=\mathbf{R}_{1}^{(l)} \mathbf{t}_{1, k}+\mathbf{v}_{1}^{(l)} \text { and } \mathbf{t}_{2, k}^{(l)}=\mathbf{R}_{2}^{(l)} \mathbf{t}_{2, k}+\mathbf{v}_{2}^{(l)},
$$

where $\mathbf{R}$ represents a rotation tensor and $\mathbf{v}$ is a translation vector. These transformations, which can be calculated reliably using the method of Veldpaus et al. (1988), are subsequently applied to the nodal coordinates $\mathbf{p}_{1, r}$ and $\mathbf{p}_{2,5}$ to realign $S_{1}$ and $S_{2}$ into the position of load configuration $(l)$. The procedure may be repeated for any load configuration.

Contact measurements. The mathematical method used to estimate joint contact areas using precise anatomical data obtained from SPG is described in this section. First, it is assumed that the geometric models of the surfaces have been rotated and translated in space to assume the relative position of the real surfaces in the intact joint under a particular load configuration. Since cartilage surfaces deform at points of contact, a criterion of contact which applies to nondeforming mathematical surfaces constructed from the SPG data must be established. Scherrer et al. (1979) defined contact areas between mathematical surfaces as all the points on one articular surface (the 'shooting surface') which fall within a specified distance $\delta$ from the other surface (the 'target surface'). In their study, the distance between the surfaces was measured along directions normal to the shooting surface. In the present study, the definition of closest distance from a known point on one surface to the other surface is modified to represent the distance from nodes of the shooting surface along a direction normal to the target surface, as described below. This definition is consistent with the strict measurement of shortest distances between points and surfaces (Mortenson, 1985).

To quantify contact areas using a proximity criterion, let the two joint surfaces be defined as primary and secondary, say $S_{1}$ and $S_{2}$, respectively (Fig. 2). The following analysis determines the regions of contact on surface $S_{1}$. The equation of a bicubic patch $B_{m}$ of surface $S_{2}$ (where $m=1-N_{2}$, the total number of patches on $S_{2}$ ), is given by $\mathbf{x}(u, w)$. The unit vector normal to the surface at $\mathbf{x}(u, w)$ is given by $\mathbf{n}(u, w)=\left(\mathbf{x}_{u}\right.$ $\left.\times \mathbf{x}_{w}\right) /\left|\mathbf{x}_{u} \times \mathbf{x}_{w}\right|$, where $\mathbf{x}_{u}=\partial \mathbf{x} / \partial u$ and $\mathbf{x}_{w}=\partial \mathbf{x} / \partial w$ are the tangent vectors at $x$, along the $u$ - and $w$-directions, respectively. The unit normal $\mathbf{n}$ is defined such that it points outward to the joint surface. The distance from a point $x$ on $B_{m}$ to a fixed point $\mathbf{p}$ (e.g. a node) on $S_{1}$ is given by $\mathbf{r}=\mathbf{p}-\mathbf{x}$. The purpose of this analysis is to find the point $\mathbf{x}$ which is closest to $p$, i.e. the vector $r$ should be normal to $S_{2}$. Mathematically, this is equivalent to having $r=t n$, where $t$ is the algebraic value of the distance between $p$ and $\mathbf{x}$ (Fig. 2). A negative value of $t$ indicates that $p$ is located in the direction pointing opposite to n, which means that $S_{1}$ overlaps $B_{m}$ (and therefore, $S_{2}$ ) at $p$ (Fig. 3). The solution to the problem of finding the minimum distance from $p$ to $B_{m}$ reduces to finding the values of $u, w$, and $t$ which satisfy

$$
\mathbf{p}-\mathbf{x}(u, w)-t \mathbf{n}(u, w)=0 .
$$

The vector equation (2) consists of three nonlinear algebraic equations which can be solved simultaneously for the three unknowns using, for example, an iterative numerical scheme wherc the Jacobian of the system is evaluated by the finite difference method. To find a valid solution, a good initial guess for $u, w$, and $t$ should be provided to the iterative solution scheme of equation (2). Such an initial guess may be obtained by first approximating each bicubic patch $B_{m}$ with two triangular planar patches. Since, for a planar patch, $\mathbf{x}(u$, $w$ ) is a linear function of $u$ and $w$, and $\mathbf{n}$ is constant, the system

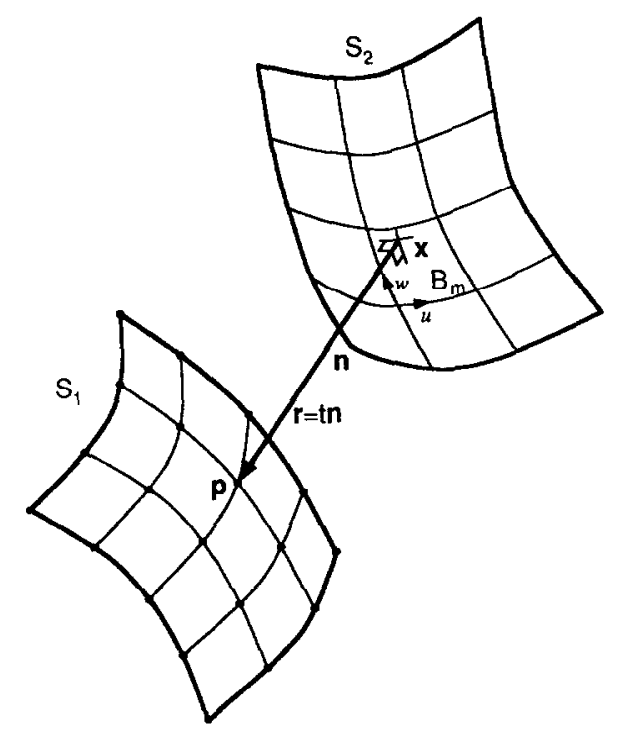

Fig. 2. Contact areas on the primary surface $S_{1}$ are defined as regions of closest proximity to the secondary surface $S_{2}$. The distance from a node $p$ of $S_{1}$ to any point $\mathbf{x}$ on $S_{2}$ is given by the length of the vector $r=p-x$. Point $x$ on $S_{2}$ is closest to $p$ when $\mathbf{r}$ is perpendicular to $S_{2}$, or more specifically, when $\mathbf{r}$ is perpendicular to some patch $B_{m}$ of the composite surface $S_{2}$.

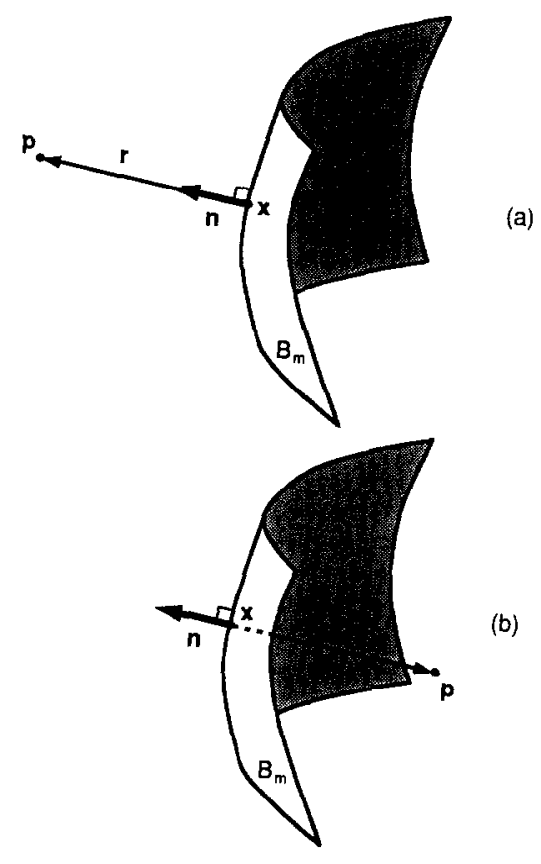

Fig. 3. The node $p$ is 'in front' of $B_{m}$ when $r=t n$ and $n$ point in the same direction $(t>0)$. When $t<0$, $p$ is 'behind' $B_{m}$, i.e. the primary surface overlaps the secondary surface at that node.

in equation (2) becomes linear in the variables $u, w$, and $t$, and can easily be solved using Gauss elimination. This procedure provides an initial estimate which can later be used for the nonlinear iterative solution (Ateshian, 1991).

A converged solution of equation (2) which yields values of $u$ and $w$ outside of the interval $[0,1]$ is rejected since it does not belong to the domain of definition of $B_{m}$. Note that the solution procedure is repeated for each patch $B_{m}$ of $S_{2}$. Three 


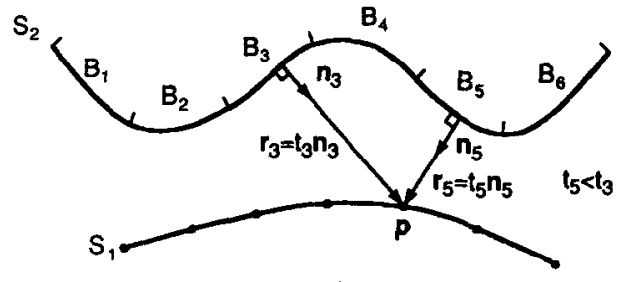

(a)

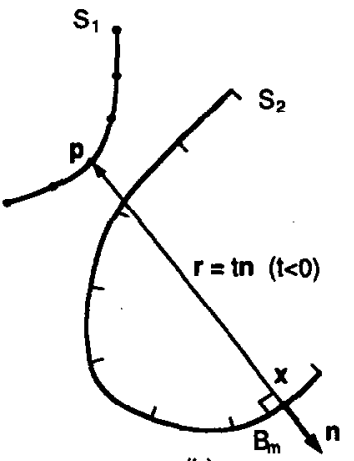

(b)

Fig. 4. (a) For a given node p on $S_{1}$, there may be multiple patches $B_{m}$ on which some point $x$ satisfies the solution $\mathbf{r}=\mathbf{p}$ $-x=t n$; in such cases, the smallest value of $t$ is chosen as the solution. (b) Under certain conditions, a point $x$ with a negative $t$ is found, while the surfaces do not overlap. These solutions are eliminated, see text.

cases may occur for any given p on $S_{1}$ : (1) no solution is found. This case typically occurs when p is located far from the 'contact areas'; (2) a single valid solution is found, i.e. a valid point $x$ was found on one patch $B_{m}$ of $S_{2} ;(3)$ more than one solution is found, i.e. there are several patches $B_{m}$ on $S_{2}$ for which a valid point $\mathbf{x}$ is found. In this case, an example of which is shown in Fig. 4(a), only the solution which yields the smallest algebraic value of $t$ is kept. A situation may arise where $t$ is negative even though the surfaces do not overlap at that particular node $p$ [Fig. 4(b)]. To avoid the selection of such a case as a valid solution, a lower bound $t_{\mathrm{ov}}$ on the value of $t$ is set; thus, any solution which yields values of $t$ algebraically smaller than $t_{\mathrm{ov}}$ is considered invalid. This lower bound may be determined from the size of the joint and the maximum expected overlap. For example, if the cartilage thickness in a joint does not exceed $2 \mathrm{~mm}$, then $t_{\text {oy }}$ may be set at $-2 \mathrm{~mm}$. Note that the above analysis does not account for the possibility of multiple valid solutions within a single patch $B_{m}$. However, this case is highly unlikely to occur since the bicubic patches are small and smooth relative to the articular surface.

A computer program was written to search for a valid solution $\left(u_{r}, w_{r}, t_{r}\right)$ for each node $p_{1, r}\left(r=1-n_{1}\right)$ according to the scheme described above. The results of the above procedure consist of two arrays of length $n_{1}$, one containing a logical value which indicates whether a valid solution was found for node $p_{1, n}$ and the other containing the distance $t_{r}$ from $p_{1, r}$ to $S_{2}$ when it was found. The value of $t$ for points inside a patch can be evaluated in the same way as for the corner nodes, although this would require prohibitively expensive numerical computations. Since patch sizes are small relative to the total joint area, it suffices to interpolate $t$ values linearly from the corner nodes to points inside the patch.

The minimum value of $t_{r}$ over all $r$ is denoted by $t_{\mathrm{min}}$. If $t_{\min }$ is positive, it approximates the closest distance between $S_{1}$ and $S_{2} ;$ if it is negative, it approximates the greatest amount of overlap between the two surfaces (Fig. 5). A proximity index, $\delta \geqslant 0$, is then defined such that all nodes $p_{1 . r}$ of $S_{1}$ for

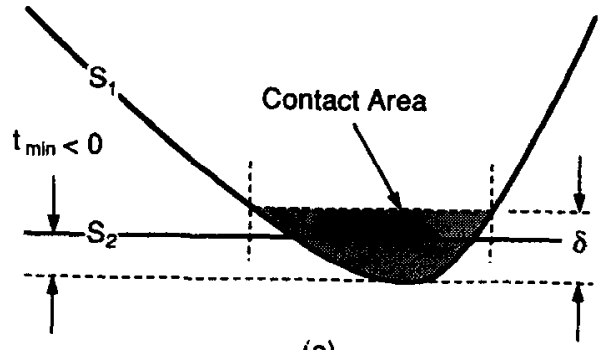

(a)

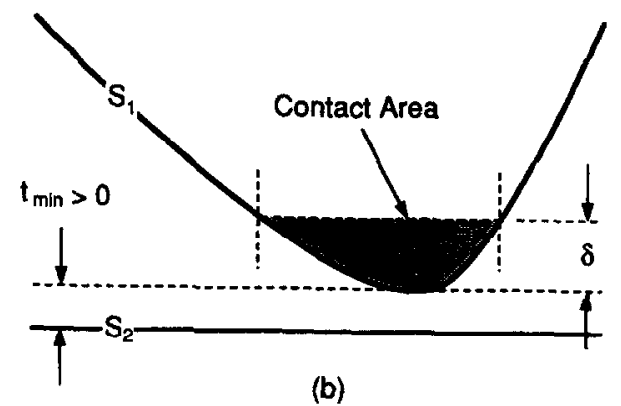

Fig. 5. Contact areas between two undeformable mathematical surfaces $\left(S_{1}, S_{2}\right)$ are defined as those areas on $S_{1}$ which fall within a prescribed distance $\delta$ from the point of closest approach of the surfaces $\left(t_{\text {min }}\right)$.

which $t_{\min } \leqslant t_{r} \leqslant t_{\min }+\delta$ are considered to be in contact with $S_{2}$ (Fig. 5). Thus, 'contact areas' are actually regions of closest proximity as determined by the parameter $\delta$. These contact regions can then be displayed in shades of gray, ranging from black for $t_{r}=t_{\min }$, to white for $t_{r} \geqslant t_{\min }+\delta$. Thus, a gray level corresponds to all those points on $S_{1}$ whose distance $t$ to $S_{2}$ fall in the range $t_{\min }+\delta_{i} \leqslant t \leqslant t_{\min }+\delta_{i+1}$, where $\delta_{i}=(i / n) \delta$, $i$ $=0-n$, and $n$ is the number of desired gray levels. Since $t_{\min }$ varies from specimen to specimen, it is more convenient to present the legend as $\delta_{i} \leqslant t-t_{\min } \leqslant \delta_{i+1}$. Note that $\delta$ is a postprocessing parameter which determines the amount of information displayed, and is not involved in the calculation of $t_{r}$. An upper bound for the value of $\delta$ can be obtained by estimating the amount of cartilage deformation under load, which in turn is limited by the cartilage thickness for that specific joint. A lower bound for $\delta$ may be chosen as the measurement uncertainty of the joint surface data (Scherrer et al., 1979).

\section{Fuji pressure-sensitive film}

Fuji Prescale Ultralow pressure-sensitive film (0.5-2.5 $\mathrm{MPa}$ ) was also used to measure contact areas in this study (e.g. Brown et al., 1991; Fukubayashi and Kurosawa, 1980; Huberti and Hayes, 1984, 1988; Fig. 6). This film consists of two specially coated sheets of PET base, $A$ and C. Sheet A contains chemical-filled microbubbles of various sizes. When sheet $A$ is pressed against sheet $C$, some of the microbubbies burst and the chemical reacts with sheet $C$ to produce red stains. The intensity of the red stain is related to the applied pressure in a nonlinear fashion. Below a certain threshold pressure, none of the microbubbles will burst; similarly, above a saturation pressure, all of the bubbles will burst. Thus, Fuji film is designed to operate within a certain range of pressures. Owing to its high sensitivity to humidity, Fuji film is sealed from the wet testing environment in joints using, for example, polyethylene sheets. Typically, under these conditions, the thickness of the film ranges from 200 to $300 \mu \mathrm{m}$. 


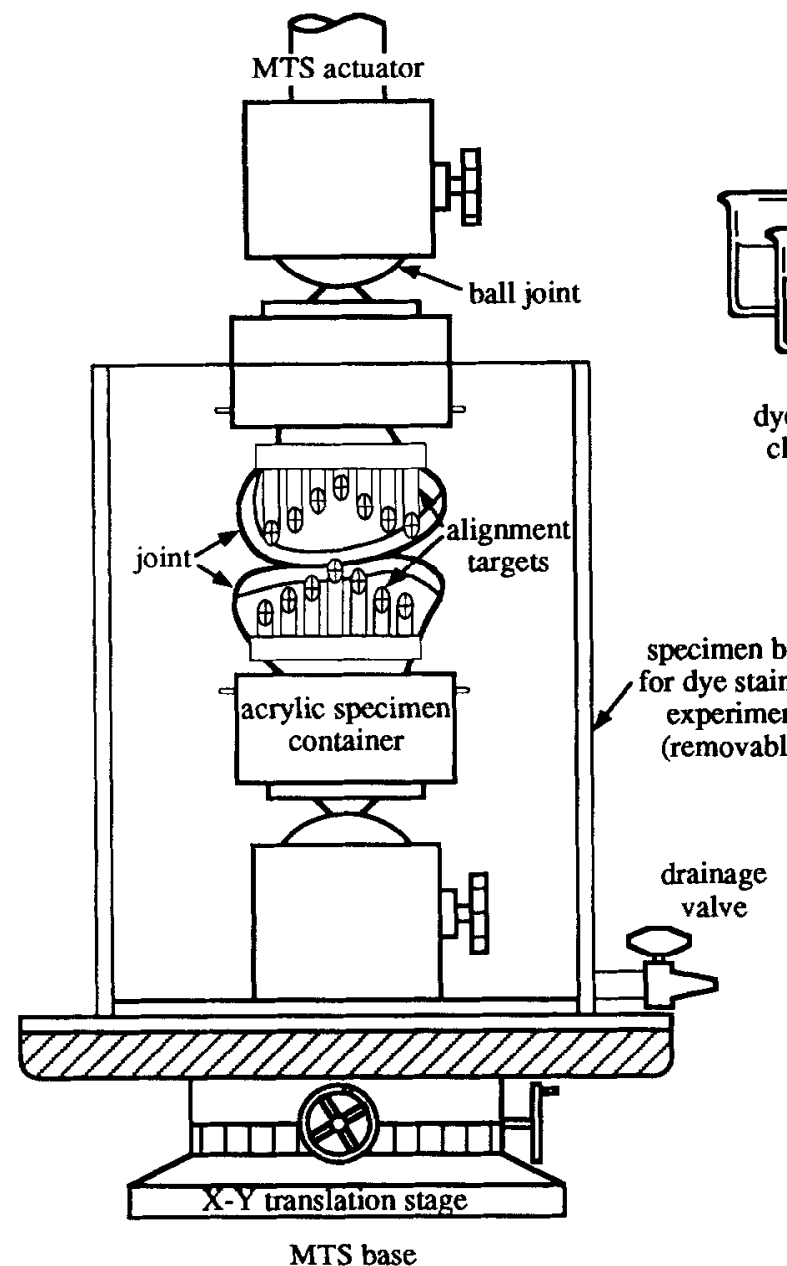

$0.01 \mathrm{M} \mathrm{K}_{4} \mathrm{Fe}(\mathrm{CN})_{6}$

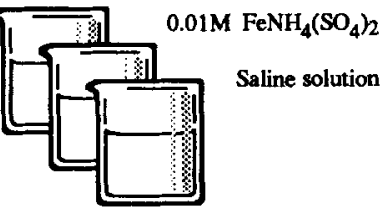

dye staining

chemicals
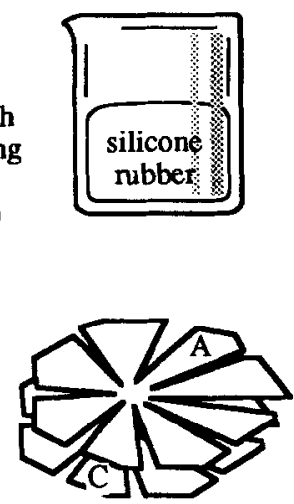

Fuji film

Fig. 6. Schematic of the loading apparatus and contact measurement methods: SPG, Fuji film; silicone rubber casting; dye staining. The specimen bath can be unscrewed at its base without altering the contact configuration.

\section{Dye staining}

Several other techniques were used to provide comparisons with the SPG contact method. The reversible dye staining method, described by Black et al. (1981), was employed and is summarized as follows: (1) the joint is bathed in saline solution; (2) immediately prior to load application, a $0.01 \mathrm{M}$ potassium ferrocyanide solution is circulated around the joint; (3) following load application, the joint is bathed in $0.01 \mathrm{M}$ ferric ammonium sulfate, which reacts with potassium ferrocyanide to produce a blue stain on the regions of cartilage which are exposed to the solution; (4) the joint is washed with saline prior to removal of the load; and (5) the staining pattern is carefully photographed and quantified, and subsequently removed by circulating $0.1 \mathrm{M}$ sodium carbonate around the joint (Fig. 7).

\section{Silicone rubber casting}

In this method, silicone rubber (Dow Corning, 3110 RTV4) in liquid form is poured around one bone of the joint, followed immediately with load application (Stormont et al., 1985). The joint is then kept under load until the silicone rubber sets (setting time $=\mathbf{4} \mathrm{min}$ ). After removal of the load, contact areas can be determined from the location and size of holes in the rubber cast (Fig. 7).

\section{MATERIALS AND TESTING PROTOCOL}

Bovine joints were used for comparing the various contact measurement techniques in this study. To test these techniques under typical conditions of incongruent contact, the lateral compartment of three menisectomized tibiofemoral joints were selected. Similarly, congruent contact was tested using three glenohumeral joints. All the joints were stored at $-25^{\circ} \mathrm{C}$ prior to specimen preparation. Since some of the contact measurement techniques required complete dissection of the joint (e.g. silicone rubber casting), all capsular tissues were removed. After marking anatomic planes on the specimens, the lateral compartments of the tibiofemoral joints were excised using a bone saw. Similarly, the glenoid was separated from the scapula. For all the joints, each bone was fixed inside a hollow acrylic tube (radius $=5.3 \mathrm{~cm}$, height $=6.4 \mathrm{~cm}$ ) using $2.4 \mathrm{~mm}$ Steinmann pins and PMMA, and anatomical markings were duplicated on the external wall of the tubes. Following these preparations, the specimens were frozen again at $-25^{\circ} \mathrm{C}$ until testing.

Joint loading was performed on a MTS computer-controlled testing machine, under load-control mode. The joint was mounted on the testing machine using spherical joints and an $X-Y$ translation stage to provide precise and redundant control for joint positioning (Fig. 6). For the tibiofe- 
moral joints, load was increased linearly from 0 to $200 \mathrm{~N}$ over a period of $60 \mathrm{~s}$, then kept constant for $300 \mathrm{~s}$, and decreased linearly down to $0 \mathrm{~N}$ over $60 \mathrm{~s}$. The same loading pattern was used for the glenohumeral joints, but with a peak load of $400 \mathrm{~N}$ to ensure that the contact pressure would exceed the threshold level of the Fuji film. Each joint underwent a testing sequence which included all of the contact measurement techniques. For tibiofemoral joints, the sequence of contact area determination went as follows: (1) A rectangular sheet of pressure-sensitive Fuji film was secured with adhesive tape to the bottom (femoral) surface, and load was applied as described above. After load removal, the A sheet was carefully removed from the surface so as not to disturb the red-stained $\mathrm{C}$ sheet, which was subsequently photographed in situ and removed. (2) Silicone rubber was poured around the bottom surface immediately prior to load application. The $360 \mathrm{~s}$ period preceding load removal provided sufficient time for the rubber to set. At the end of the loading sequence, the precise position of the closely fitting rubber cast was marked relative to the two bones of the joint for future reference, and the cast was removed. (3) The dye staining technique was applied under the same load configuration. The blue stain on the cartilage surface was not yet removed at this stage. (4) The SPG calibration frame was placed in front of the alignment targets such that the coordinate axes of the frame were aligned with the anatomical planes of the specimen, as indicated from the acrylic tube markings. The joint was loaded and photographed (approximately $330 \mathrm{~s}$ after load application) using the SPG apparatus. The corresponding stereogram displayed the two sets of surface alignment targets, within the SPG calibration frame. (5) The Fuji film method was repeated, using a carefully cut film which closely conformed to the joint surface. The $\mathrm{C}$ sheet remained secured to the surface at the end of this testing phase.

The glenohumeral joints were tested in a similar manner, but with steps 2 and 3 reversed. In order to compare precisely the results of each measurement technique in this testing sequence, all of the contact area data were quantified relative to the joint articular surfaces using the SPG system as follows: (a) The Fuji film contact patterns were delineated directly on the 'conforming' $C$ sheet (which was still secured to the joint surface) by drawing black dots around the contact area contours [Fig. 7(a)]. A stereogram of the joint was then obtained in order to quantify the three-dimensional coordinates of these contour dots relative to the alignment targets of the surface. The contact patterns on the nonconforming rectangular sheets, as described in step 1 above, were not quantified. (b) After removal of the $\mathrm{C}$ sheet, the silicone rubber cast was closely fitted back onto the surface of the joint, making use of prior markings. Black dashes were drawn on the inner edge of all the holes appearing in the cast, which corresponded to the contact areas for this technique [Fig. 7(b)]. Then the joint, with the silicone cast, was photographed with SPG to quantify similarly the threc-dimensional coordinates of the inner edge of the black dashes relative to the alignment targets. This step was performed for both surfaces of the joint. (c) At this stage, the blue stain from the dye staining method was still present on both cartilage surfaces of the joint. Once again, black dots were drawn, this time directly on the cartilage surfaces, along the periphery of the various unstained contact zones [Fig. 7(c)]. The threedimensional coordinates of these dots were also quantified for both joint surfaces with SPG. (d) Finally, the blue stains were chemically removed, and the entire articular surfaces were quantified, relative to their respective alignment targets using SPG [Fig. 7(d)]. All the contact areas are displayed on this SPG-determined geometric model of the joint [Fig. 7(d)].

\section{RESULTS}

The contact contours of the dye staining, silicone rubber casting and Fuji film methods were superposed on the contact areas determined from SPG using the alignment targets. Figure 8(a)-(c) shows the contact areas on all three femoral surfaces of the tibiofemoral joints, while Fig. 9 (a)-(c) shows the results for all three humeral heads from the shoulder joints.

In all the samples except one shoulder joint [Fig. 9(c)], the dye staining method yielded the largest contact areas. This result may be attributed to surface tension effects in the ferric ammonium sulfate solution used for dye staining (Bullough, 1974). Specifically, surface tension would prevent the solution from seeping into the narrow regions near the periphery of the actual contact areas. Thus, in general, the dye staining method tends to slightly overestimate the size of the contact areas in joints. In one of the glenohumeral joints [Fig. 9(a)], contact was detected by the dye staining technique in a region which was not observed to be in contact using any of the other measurement methods (area A). It is suspected that an air bubble, which may have remained trapped between the glenoid and humeral surfaces when the joint was submerged in solution, could have prevented the staining solution from reaching this region. For a congruent joint such as the shoulder, a small air bubble may spread over a large area when squeezed between the opposing surfaces. In another shoulder joint [Fig. 9(b)], by comparing dye staining data with SPG results, it appears that a noncontacting region which was isolated within a larger contacting region could not be reached by the dye (area B). Thus, the isolated 'island' was erroneously determined to be in contact.

Silicone rubber casting yielded smaller contact areas than dye staining. This difference in size was more significant in all the glenohumeral joints, as well as in one of the tibiofemoral joints [Fig. 8(a)]. These results demonstrated that certain regions that were in contact according to dye staining. SPG and Fuji film methods were actually covered by a thin silicone rubber layer. Thus, by the standard used for determining contact areas with this technique, these regions were not in contact (e.g. Fig. 9(b) shows a contact pattern for silicone rubber in the superior aspect of the humeral head only).

In general, Fuji film produced the smallest contact areas when compared to dye staining and silicone rubber casting techniques. Using a rectangular sheet yielded satisfactory results in the incongruent tibiofemoral joints, but resulted in significant crimping of the Fuji film in the congruent glenohumeral joints (Fig. 10).

The SPG contact data exhibited remarkable similarities with the results from the other techniques. The most significant feature of the SPG results was the ability to predict the contact contours of the other methods by looking at various proximity levels on the SPG contact area map (Figs 8 and 9). Thus, in general, the dye staining, silicone rubber casting and Fuji film data were fitted within contours of constant proximity, in a decreasing sequence of $\delta$ values. Furthermore, grayscalc intensities on the SPG contact maps were always in close agreement with red color intensities of the Fuji film. This observation confirms that regions of higher contact pressures correspond to regions of closer proximity.

\section{DISCUSSION}

The objective of this study was to introduce the methodology of the SPG contact technique, and compare the performance of this technique to other contact methods commonly used in biomechanics of diarthrodial joints. The comparison of various techniques is an especially difficult task since it involves the comparison of the shape, size, and location of simply or multiply connected contact regions on surfaces of complex topography. While the surface area of these contact regions may he quantified by a simple scalar quantity, shape and location are not so easily quantified. Therefore, a scalar quantitation of the relative performance of the various contact measurement methods is not practical 
Fuji Film

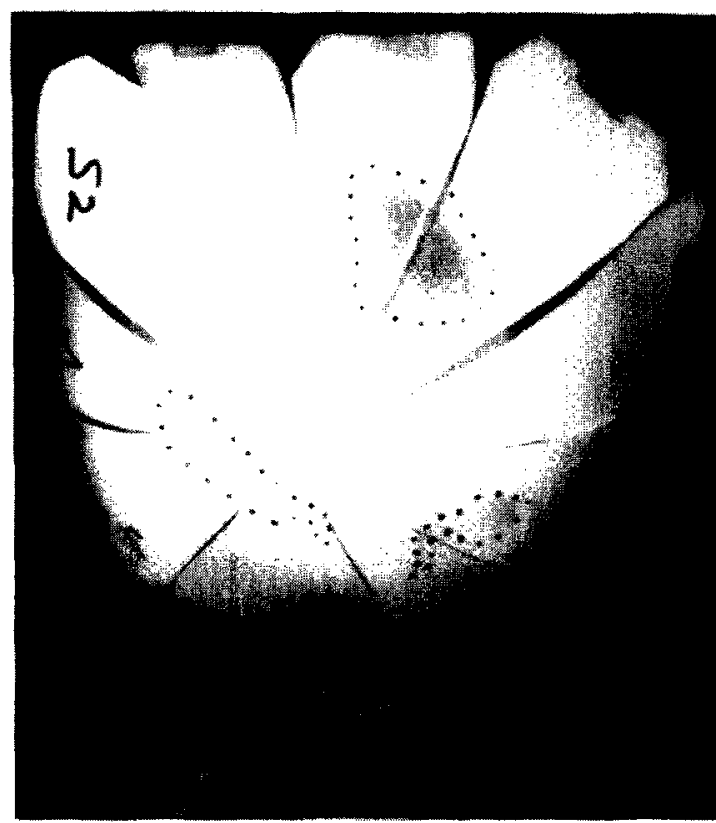

Alignment

Targets

(a)

\section{Silicon Rubber}

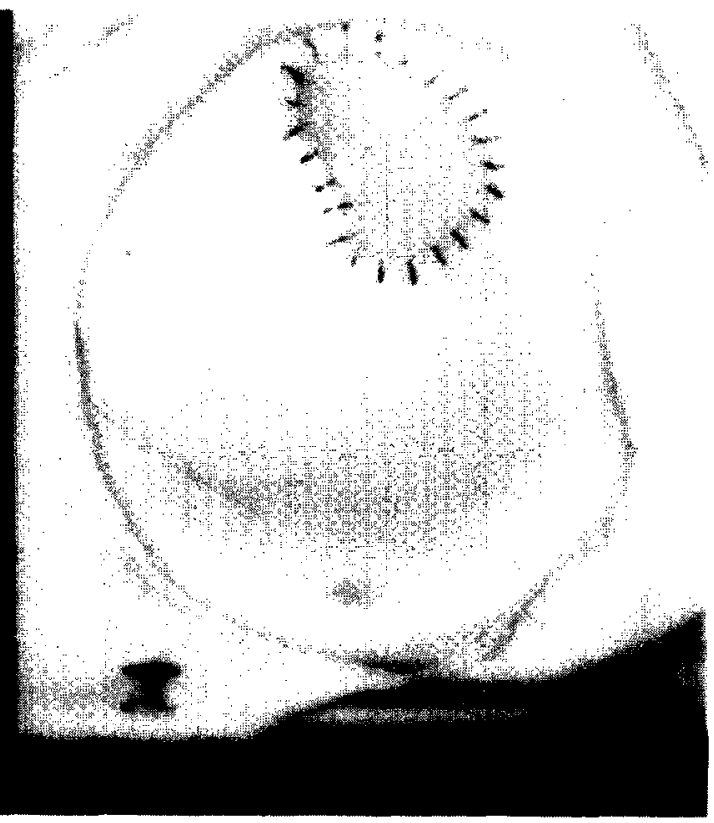

(b)

\section{Dye Stain}

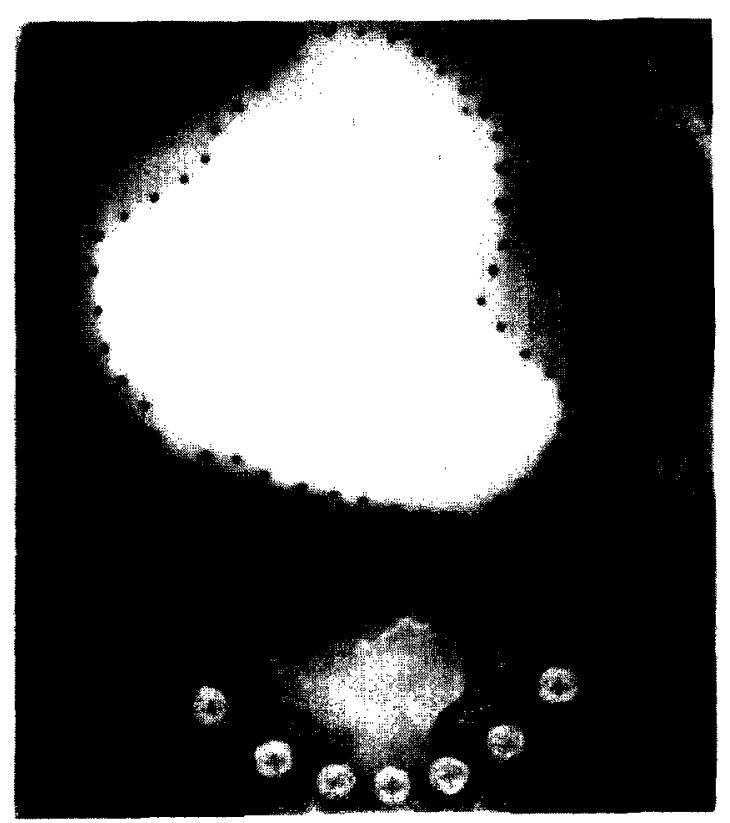

(c)
Stereophotogrammetry

$S$

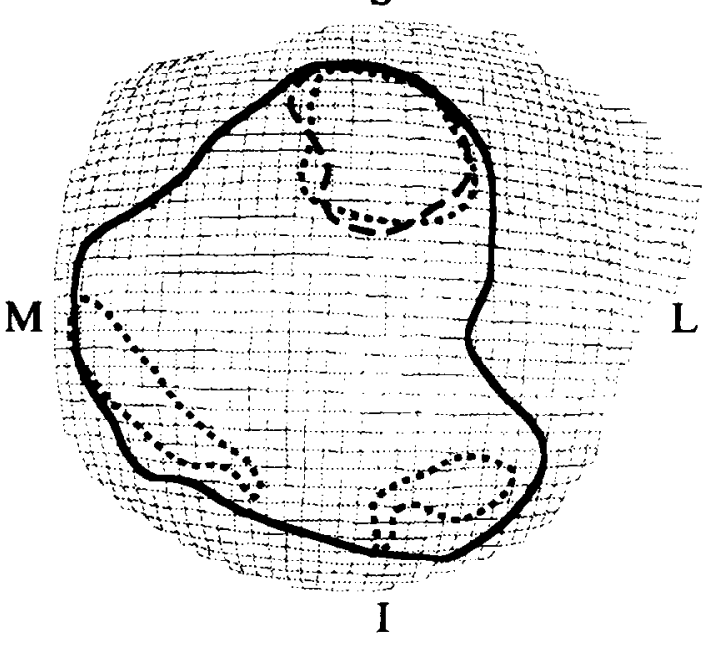

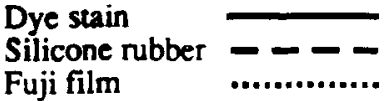

(d)

Fig. 7. The contact patterns from each technique were marked with black dots or dashes for threedimensional SPG analysis: (a) Fuji film; (b) silicone rubber; (c) dye staining. These contact patterns were subsequently superposed on the SPG geometric model of the joint articular surface for direct comparison (d). 


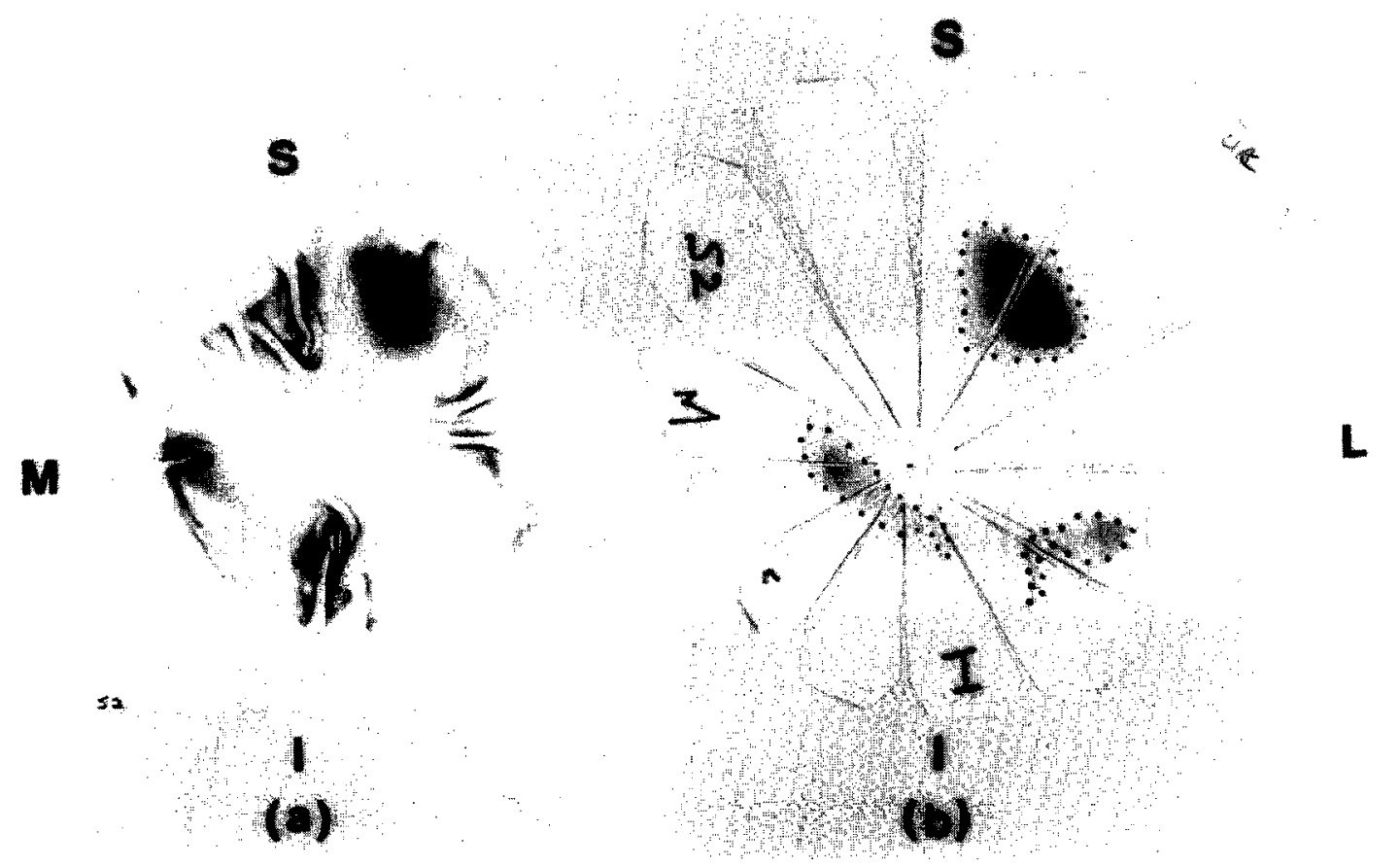

Fig. 10. (a) A rectangular sheet of Fuji film results in significant crimping in the congruent glenohumeral joints. (b) More reliable results are obtained when the Fuji film sheets are cut to conform to the articular surfaces. The dotted contour shown on this sheet corresponds to the Fuji film data of the specimen in Fig. 8(b). 
or even feasible. In this study, a graphical quantitation of the contact areas was performed using SPG, where three-dimensional computer representations of the various results were projected and superposed onto geometric models of the articular surfaces. Thus, the outcome of all four methods could be compared directly from these quantitative threedimensional graphical results.

The SPG contact method displays contours of constant proximity for the two opposing surfaces of a joint. Each contour corresponds to a specific proximity index $\delta$, and a typical plot of the SPG contact data displays the contours for a range of discrete values of $\delta$. In theory, using thin-layer elastic or viscoelastic contact analysis of smooth surfaces (Armstrong, 1986; Johnson, 1985), the area contained within each contour of proximity $\delta$ will be the contact area when the maximum deformation (i.e. approach) for that particular load is equal to $\delta$. For diarthrodial joints, the maximum deformation is generally not known experimentally. How. ever, the upper asymptotic bound on this value may be assumed to be the maximum cartilage thickness in the region

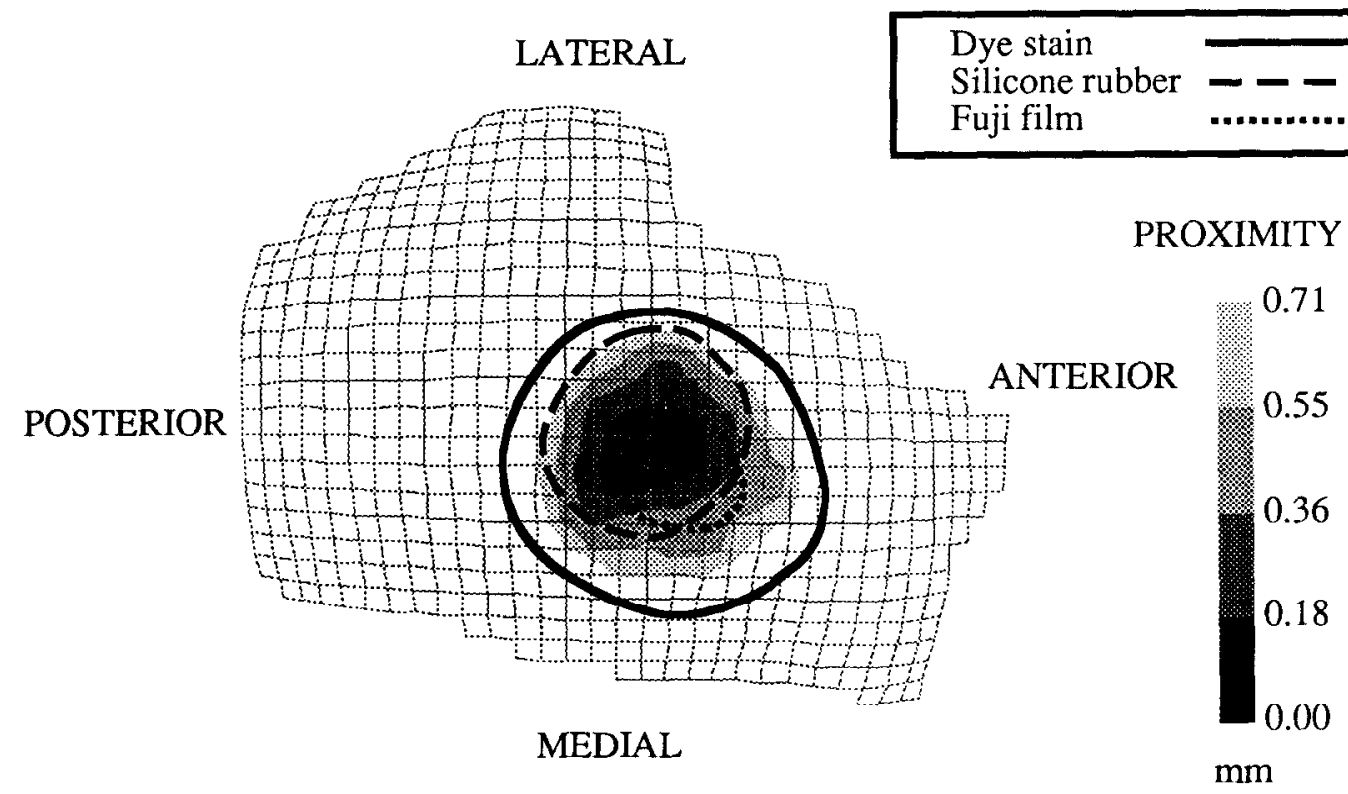

(a)
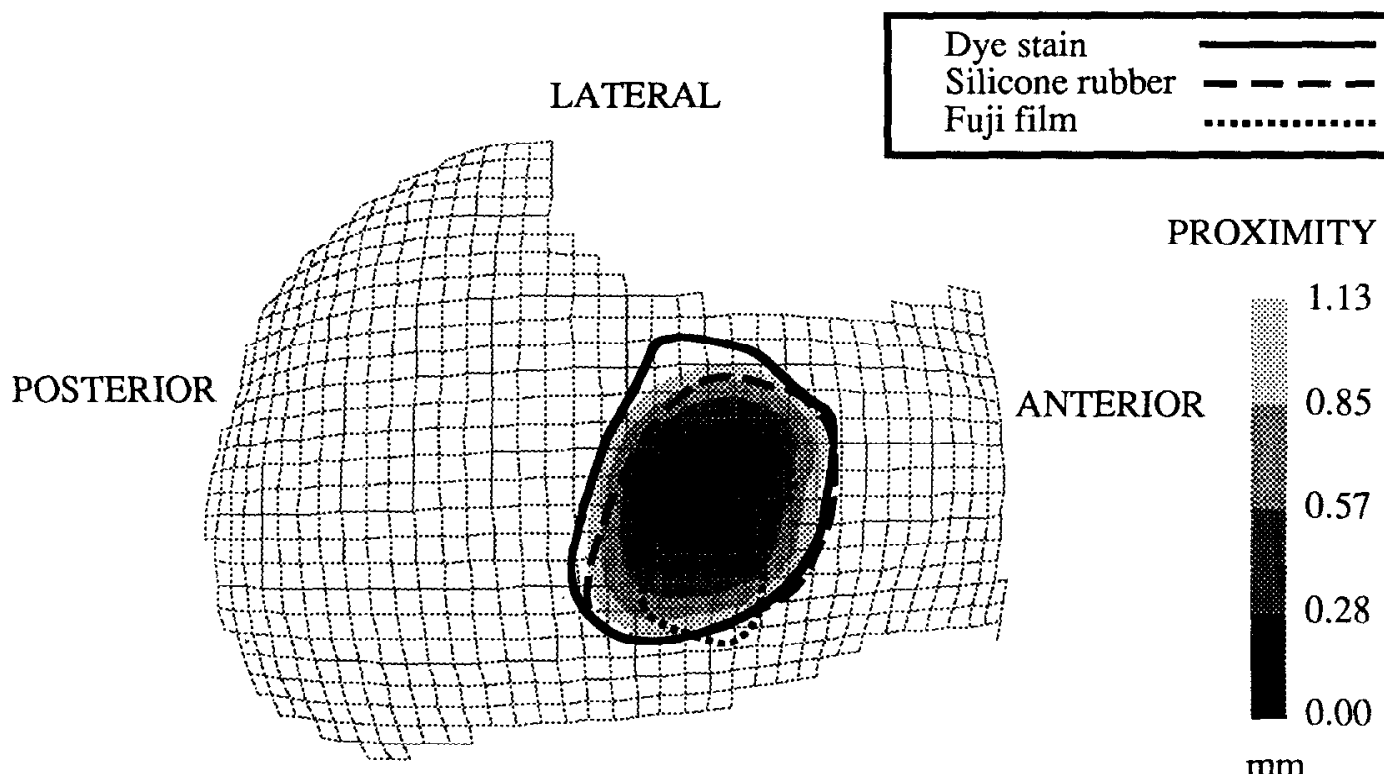

MEDIAL

PROXIMITY

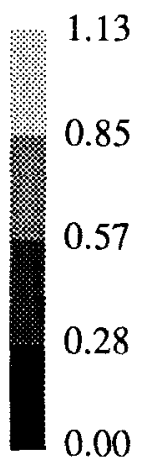

$\mathrm{mm}$

(b)

Fig. $8(\mathrm{a}, \mathrm{b})$. 


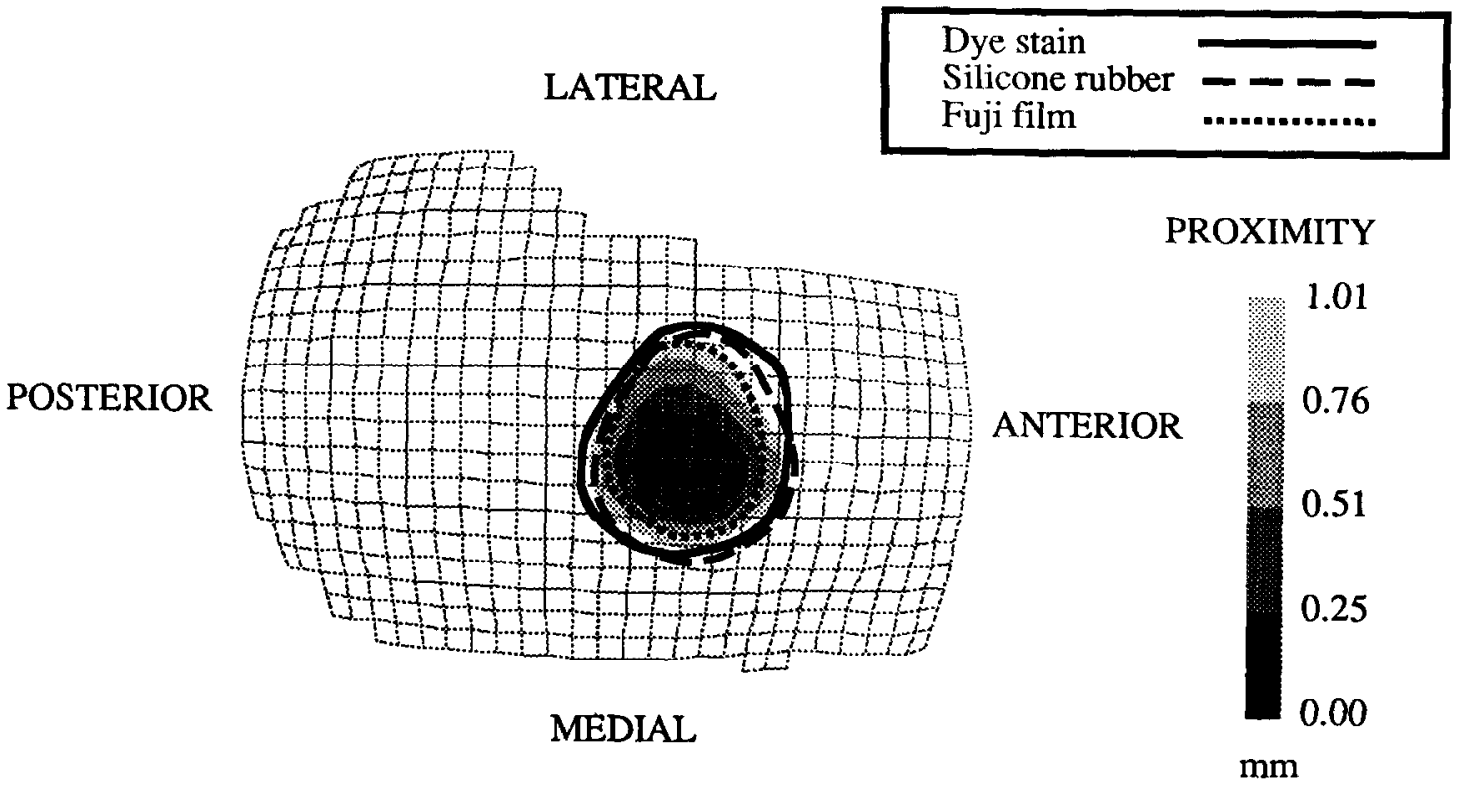

(c)

Fig. 8. Contact areas on the femoral surface of all three tibiofemoral joints. The gray shaded regions represent the proximity contours of the SPG contact data, while the solid, dashed and dotted contours correspond to the dye staining, silicone rubber casting, and Fuji film contact area data, respectively.

of contact. Thus, by varying $\delta$ between zero and this limiting upper bound, the SPG contact method will provide the full spectrum of possible contact areas with increasing cartilage deformation for a particular joint position. In this study, the proximity index $\delta$ for the SPG contact area data was obtained from the approach of the articular surfaces as measured by the MTS for each joint. This choice yields a reasonable approximation of total deformation of the two cartilage surfaces provided that the direction of loading is parallel to the direction of normal approach of the surfaces, and other deformations within the system (i.e. bone) are negligible.

The silicone rubber contact results indicate that this method yields smaller contact areas than the dye staining method and, in most cases, the SPG method as well. Indeed, the silicone rubber technique may be viewed as a squeeze-film effect, where liquid silicone is driven out of the region of contact as the two articular surfaces approach each other. From basic lubrication theory, it is known that the silicone 'film' cannot be entirely squeezed out within a finite time period. This is further complicated by the fact that silicone turns from liquid to solid over a short time period, thus increasing its viscosity in a nearly exponential way. A conclusion from these observations is that silicone rubber casting will tend to underestimate the size of actual contact areas, or even fail to detect contact in some regions of a joint. Obviously, this problem is more significant in congruent joints [e.g. Fig. 9(b) and 9(c)]. Note that this result appears to contradict the findings of Stormont et al. (1985), who reported that silicone rubber overestimates the contact areas. The cause for this contradiction is not clear.

In all of the joints, Fuji film gave consistent results, i.e. it did not predict contact in regions for which contact was not detected by any of the other methods. However, in some cases, while the contact contours determined from all other techniques appeared nearly concentric to one another, the Fuji film contact pattern was slightly offset [e.g. Fig. 8(a) and (b)]. This result may be attributed to a combination of several factors: (a) the finite thickness of the Fuji film slightly alters the topography of the articular surfaces, thereby altering the contact areas; (b) if contact occurs along surfaces which are inclined to the direction of approach, some sliding of the $\mathrm{A}$ sheet over the $C$ sheet may occur which will register a 'spurious' red stain due to the high sensitivity of Fuji film to shear stresses (Rudert $e t$ al, 1988); (c) the C sheet itself may slip during loading and/or unloading, so that the final position of the film at the time of data acquisition may not accurately reflect its position during contact. While (a) is an inherent problem of Fuji film, effects due to (b) and (c) may be carefully controlled, as attempted in this study, to minimize possible errors. The threshold effect in the Fuji film contact data was very apparent from the results of this study; this effect resulted in smallcr contact areas than other methods, except for silicone rubber in two of the glenohumeral joints [Fig. 9(b) and (c)]. By using the lowest threshold level available from the manufacturer, this effect was also minimized. It should also be noted that relatively small loads were applied to the joints in this study, a fact which highlights the threshold effect of Fuji film. This effect is relatively less significant when high loads and contact pressures are applied. Furthermore, the method of placing dots along the periphery of the Fuji contact regions relies on the visual perception of the investigator. While visual acuity was sufficient to perform this task in all the specimens, a digital scanning procedure (which is feasible when the film is removed from the articular surface) can provide a more precise detection of the desired threshold level. Finally, as a general rule to avoid crimping (Fig. 10), Fuji film sheets must be cut to conform to the articular surfaces as has been noted by other investigators.

The results of this study show that the precise extent of the time-dependent contact regions cannot be determined exactly by any of the static methods tested here due to inherent limitations in each of the methods. Therefore, a measure of the contact area size, as determined by any one of these methods, should always be viewed within the context of these 
limitations. Overall, for incongruent joints, the agreement between all the techniques is reasonably good (Fig. 8). When congruent joints are being tested, however, there can be significant differences in the outcome of each method. From this study, it appears that the Fuji film and SPG methods produced more similar contact results than dye staining and silicone rubber casting.
As a final discussion point, this study did not address the accuracy of the contact area determination techniques, but rather assessed their ability to produce a consistent pattern of contact areas. Determination of the accuracy of these techniques would require quantitative scales for describing shapes, size, and location of contact regions on the joint surface. Such artificial scales are less preferable than an

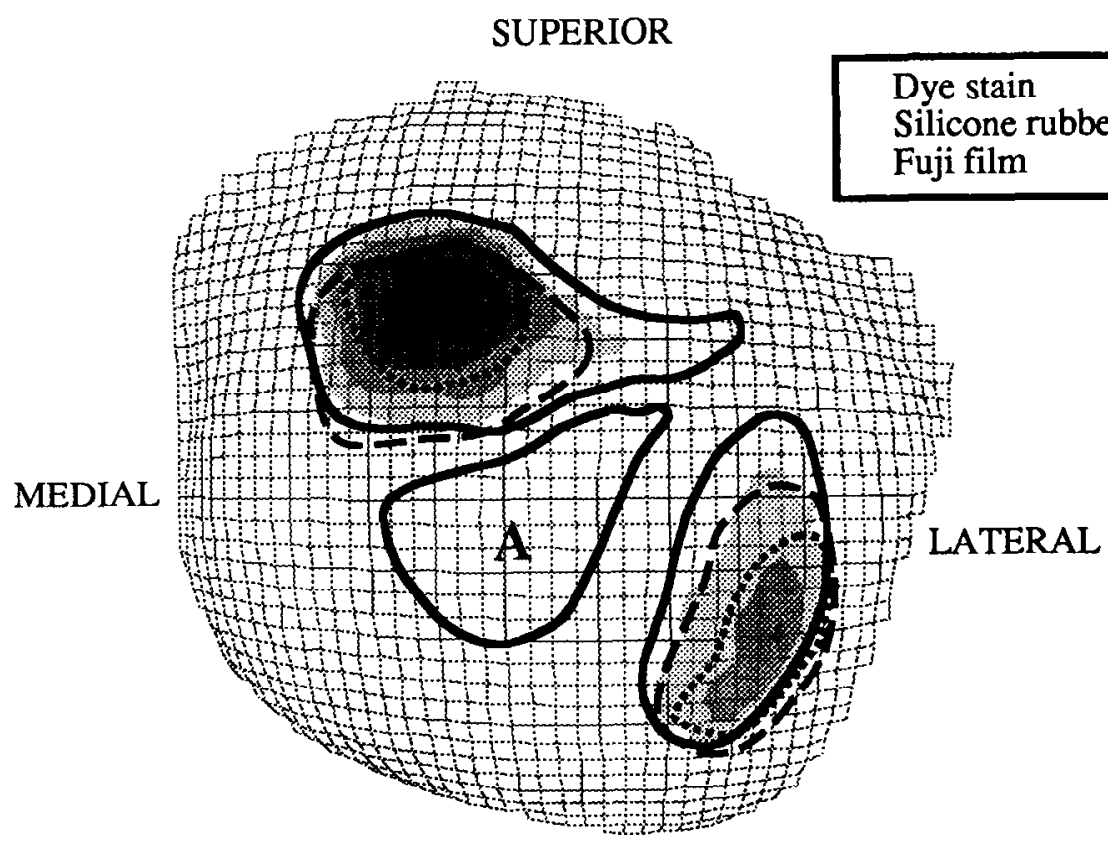

PROXIMITY

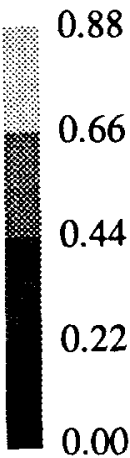

INFERIOR

(a)

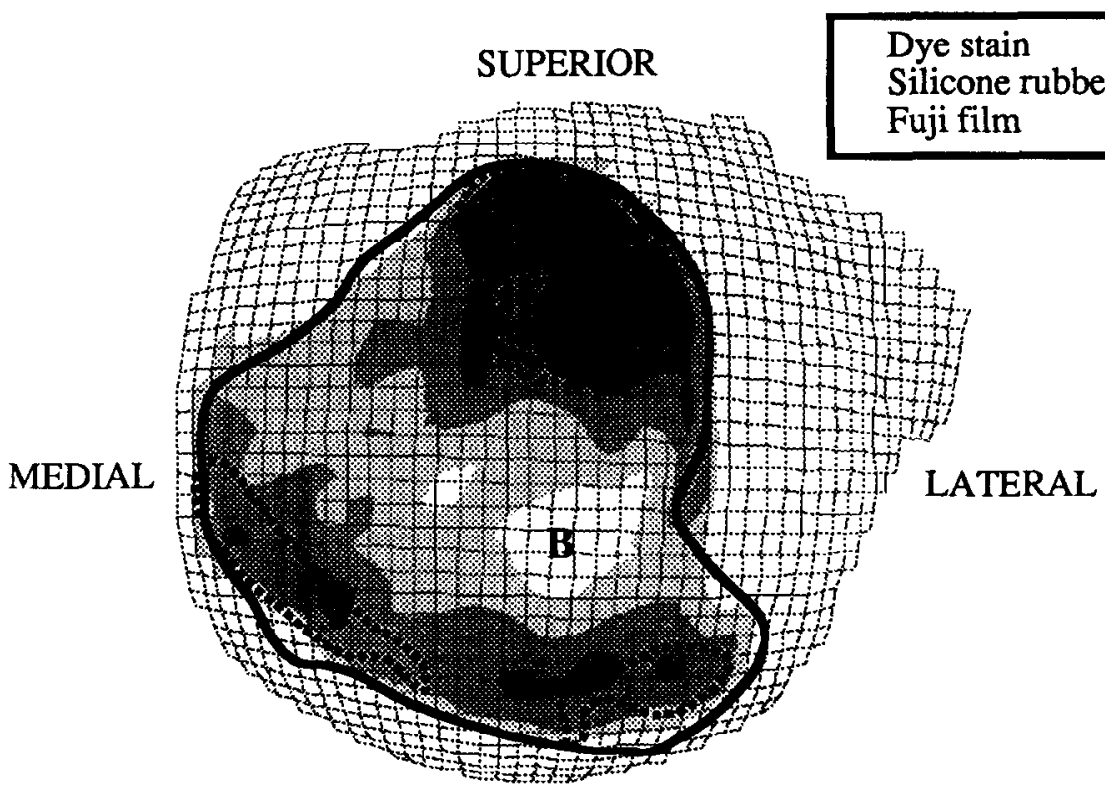

PROXIMITY

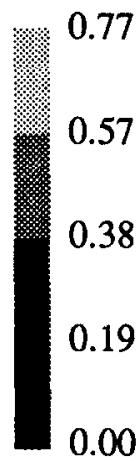

INFERIOR

(b)

Fig. 9(a,b). 


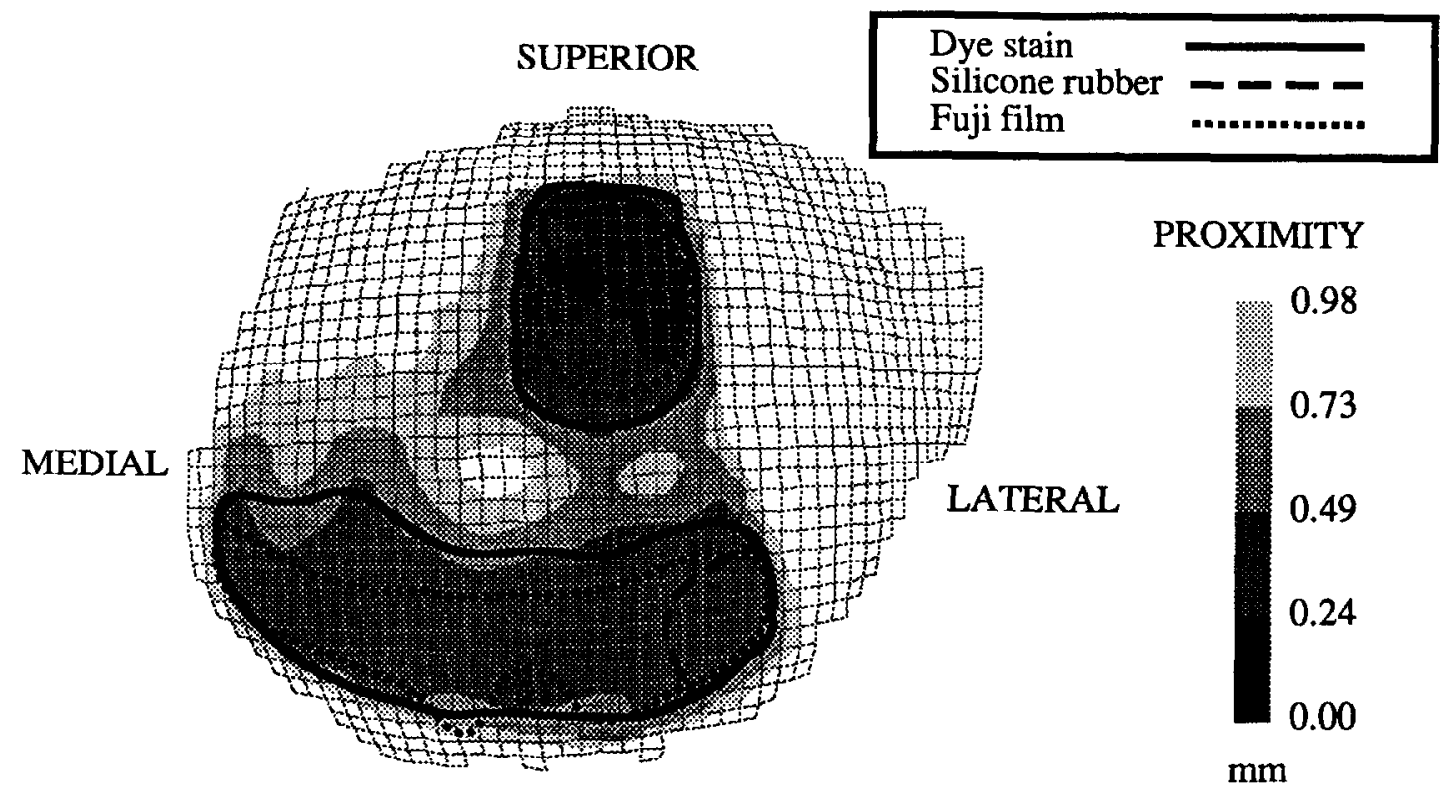

INFERIOR

(c)

Fig. 9. Contact area results for all three bovine humeral heads. 'A' indicates a region identified as a contact region by the dye staining method only. This result is believed to have been caused by a trapped air bubble. ' $\mathrm{B}$ ' indicates a noncontacting region which may be completely surrounded by contact areas. Such a region cannot be reached by the dye staining chemical and will be erroneously labeled as a contact region using that technique.

actual visualization of size, shape and location. Without a quantitative scale, a definition of a 'gold standard' measurement technique is not possible. The goal of this study was not to develop a gold standard, but rather to provide information which may help investigators in selecting or discarding a particular contact area measurement technique in light of their specific applications.

\section{CONCLUSION}

Dye staining, silicone rubber casting, Fuji film and SPG contact area determination methods were compared using congruent and incongruent bovine joints. Each method demonstrated some strengths and weaknesses. Dye staining and silicone rubber casting may provide erroneous or incomplete results under some circumstances, especially if they are used in congruent joints. Furthermore, both of these techniques require dissection of the joint capsular tissues whenever more than one load configuration is desired, in order to allow for data acquisition. For some joints, this dissection may adversely affect joint stability and thus significantly alter contact area results. In this study, Fuji film contact results appeared to be more consistent with the SPG results than those obtained by dye staining and silicone rubber casting. Fuji film, which requires careful manipulation to avoid artifacts (e.g. crimping, sliding, shear stress staining) can be expected to underestimate the contact area, especially under low loads. This limitation may be outweighed by the added ability to measure contact pressures using suitable calibrations, albeit within a preset rangc. Unlike the other techniques, however, the Fuji film method yields contact on one side of the joint only, unless the experiment is repeated for both sides for each load configuration. It is possible that the experiment could be performed using two sets of $\mathrm{A}$ and $\mathrm{C}$ sheets, secured to each of the opposing joint surfaces. However, this approach would double the thickness of the film and increase procedural difficulties.

The SPG contact area data were always consistent with those of the Fuji film, and could always be related to those dye staining and silicone rubber casting results which were considered more reliable (i.e. not artifacts). By displaying contours of constant proximity, the SPG method was also able to predict the contact regions described by the other techniques, thus providing an explanation for the observed discrepancies among the various methods. Furthermore, as opposed to dye staining and silicone rubber casting, the SPG technique provides some measure of the relative contact stress intensity within the contact regions, which can be inferred from the degree of proximity (intensity of the gray shades) on the contact area maps. However, unlike Fuji film, the SPG contact data cannot be used to predict directly contact stress values. Contact stress predictions could be obtained in the future by incorporating the precise geometric and kinematic data presented here into finite element models with appropriate constitutive models for articular cartilage (e.g. Mow et al., 1980; Spilker et al., 1990).

While dye staining, silicone rubber casting and Fuji film provide an immediate visual assessment of the contact areas, the SPG contact method requires a significant amount of data processing, including digitization of the stereograms and extensive proximity calculations. Some image processing techniques may be used to reduce considerably digitizing time in the future. However, the effort involved in the use of the SPG contact technique may be weighed against the additional merits which are summarized as follows: (1) as opposed to the other methods described above, the SPG technique does not require any violation of the joint capsular integrity, or the insertion of a device or substance into the joint space, during the phase of kinematic data acquisition; 
(2) contact areas can be determined for a multitude of joint positions and a variety of loading conditions simply by photographing the intact joint in each configuration; (3) a three-dimensional model of the joint, including both cartilage and bony geometry is created which can be saved for subsequent studies or analyses (Ateshian et al., 1992); and (4) contact areas can be superposed directly on a precise computer-generated graphical representation of the joint surfaces to provide a clear, comprehensive, and three-dimensional visualization of the experimental results. In summary, the SPG contact method offers a valuable and reliable technique for studying contact mechanics of articular surfaces in diarthrodial joints

Acknowledyement This study was supported in part by a Whitaker Foundation grant, National Institutes of Health Grant No. AR38733, and a Bristol-Myers Squibb/Zimmer Center of Excellence Grant for Orthopaedic Research.

\section{REFERENCES}

Ahmed, A. M. (1983) A pressure distribution transducer for in-vitro static measurements in synovial joints. $A S M E$ J. biomech. Engng 105, 309-314.

Ahmed, A. M. and Burke, D. L. (1983a) In-vitro measurement of static pressure distribution in synovial joints-Part I: tibial surface of the knee. $J$, biomech. Engng 105, 216-225.

Ahmed, A. M. and Burke, D. L. (1983b) In-vitro measurement of static pressure distribution in synovial joints--Part II: retropatellar surface. $J$. biomech. Engng 105, 226-236.

Armstrong, C. G. (1986) An analysis of the stresses in a thin layer of articular cartilage in a synovial joint. Engng Med. $15,55-61$

Ateshian, G. A. (1991) Biomechanics of diarthrodial joints: applications to the thumb carpometacarpal joint. $\mathrm{Ph}$. D thesis. Columbia University, New York.

Ateshian, G. A. (1993) A B-spline least-squares surface-fitting method for articular surfaces of diarthrodial joints. ASME J. biomech. Engng (in press)

Ateshian, G. A., Rosenwasser, M. P. and Mow, V. C. (1992) Curvature characteristics and congruence of the thumb carpometacarpal joint. $J$. Biomechanics 25, 591-608.

Ateshian, G. A., Soslowsky, L. J. and Mow, V. C. (1991) Quantitation of articular surface topography and cartilage thickness in knee joints using stereophotogrammetry. J. Biomechanics 24, 761-776.

Baratz, M. E., Rubak, D. C., Fu, F. H. and Rudert, M. J. (1988) Peripheral tears of the meniscus-the effect of open versus arthroscopic repair on intraarticular contact stresses in the human knee. Am. J. Sports Med. 16, 1-6.

Black, J. D., Matejczyk, M. and Greenwald, A. S. (1981) Reversible cartilage staining technique for defining articular weight-bearing surfaces. Clin. Orthop. Rel. Res. 159, 265-267.

Brown, T. D., Pone, D. F., Hale, J. E., Buckwalter, J. A. and Brand, R. A. (1991) Effects of osteochondral defect size on cartilage contact stress. J. orthop. Res. 9, 559-567.

Brown, T. D. and Shaw, D. T. (1983) In vitro contact stress distributions in the natural human hip. J. Biomechanics 16, 373-384

Brown, T. D. and Shaw, D. T. (1984) In vitro contact stress distribution on the femoral condyles. J. orthop. Res. 2 , 190-199.

Bullough, P. G. (1974) The hip. In Proc. of the 2nd Open Scientific Meeting of The Hip Society (edited by Harris, W. H.), pp. 21-22, C. V. Mosby, St. Louis.

Fukubayashi, T. and Kurosawa, H. (1980) The contact area and pressure distribution pattern of the knee. Acta orthop. scand. 51, 871-879.

Ghosh, S. K. (1979) Analytical Photogrammetry. Pergamon Press, New York.
Greenwald, A. S. and O'Connor, J. J. (1971) The transmission of load through the human hip joint. J. Biomechanics 4, 507-528.

Haut, R. C. (1989) Contact pressures in the patellofemoral joint during impact loading on the human flexed knee. J. orthop. Res. 7, 272-280.

Hodge, W. A., Carlson, K. L., Fijan, R. S., Burgess, R. G., Riley, P. O., Harris. W. H. and Mann, R. W. (1989) Contact pressures from an instrumented hip endoprosthesis. $J$. Bone Jt Surg. 71A, 1378-1386.

Howell, D. S., Treadwell, B. V. and Trippel. S. B. (1992) Etiopathogenesis of osteoarthritis. In Osteoarthritis: Diaynosis and Medical/Surgical Management (2nd Edn) (edited by Moskowitz, R. W., Howell, D. S., Goldberg, V. M. Mankin, H. J.), pp. 233-262. W. B. Saunders, Philadelphia. Huberti. H. H. and Hayes, W. C. (1984) Patellofemoral contact pressures. J. Bone Jt Surg. 66A, 715-724.

Huberti, H. H. and Hayes. W. C. (1988) Contact pressures in chondromalacia patellae and the effects of capsular reconstructive procedures. J, orthop. Res. 6, 499-508.

Huiskes, R., Kremers, J., de Lange, A., Woltring, H. J., Selvik, G. and van Rens, Th. J. G. (1985) Analytical stereophotogrammetric determination of three-dimensional knee-joint geometry. J. Biomechanics 18, 559-570.

Johnson, K. L. (1985) Contact Mechanics. Cambridge University Press, Cambridge.

Kurosawa, H., Fukubayashi, T. and Nakajima, H. (1980) Load-bearing mode of the knee joint, physical behavior of the knee joint with or without menisci. Clin. Orthop. Rel. Res. 149, 283-290.

Manouel, M., Pearlman, II. S.. Belakhlef, A. and Brown. T. D. (1992) A miniature piezoelectric polymer transducer for in vitro measurement of the dynamic contact stress distribution. J. Biomechanics 25, 627-635.

Mortenson, M. E. (1985) Geometric Modeling. Wiley. New York.

Mow, V. C., Kuei, S. C., Lai, W. M. and Armstrong. C. G. (1980) Biphasic creep and stress relaxation of articular cartilage in compression: theory and experiments. $J$. biomech. Engng 102, 73-84.

Rudert, M. J., Baratz, M. E.. Rehak, D. C. and Fu. F. H. (1988) Loading characteristics of Pressensor: a pressure sensitive film. Trans. orthop. Res. Soc. 34, 226.

Scherrer, P. K. (1977) Determining the distance between the surfaces of two rigid bodies using parametric surface patches and an instrumented spatial linkage. Ph.D. thesis, Purdue University.

Scherrer, P. K. and Hillberry, B. M. (1979) Piecewise mathematical representation of articular surfaces. J. Biomechanics 12, 301-311.

Scherrer, P. K., Hillberry, B. M. and van Sickle, D. C. (1979) Determining the in-tivo areas of contact in the canine shoulder. ASME J. biomech. Engng 101, 271-278.

Seedhom, B. B. and Tsubuku, M. (1977) A technique for the study of contact between visco-elastic bodies with special reference to the patello-femoral joint. .I. Biome hanic: 10, $253-260$.

Singerman, R. J., Pedersen, D. R. and Brown, T. D. (1987) Quantitation of pressure-sensitive film using digital image scanning. Exp. Mech. 27, 99-105.

Soslowsky, L. J., Flatow, E. L., Bigliani, L. U.. Pawluk, R. J. and Mow, V. C. (1992) Quantitation of in situ contact areas at the glenohumeral joint: a biomechanical study. J. orthop. Res. 10, 524-534

Spilker, R. L., Suh, J.-K. and Mow, V. C. (1990) Effects of friction on the unconfined compressive response of articular cartilage: a finite element analysis. $J$. biomech. Engny 112, 138-146.

Stormont, T. J., An, K. N., Morrey, B. F. and Chao, E. Y. (1985) Elbow joint contact study: comparison of techniques. J. Biomechanics 18, 329-336.

Tencer, A. F., Viegas, S. F., Cantrell, J., Chang, M., Clegg, P., Hicks. C., O'Meara, C. and Williamson, J. B. (1988) 
Pressure distribution in the wrist joint. J. orthop. Res. 6, 509-517.

Veldpaus, F. E., Woltring, H. J. and Dortmans, L. J. M. G. (1988) A least-squares algorithm for the equiform transformation from spatial marker co-ordinates. J. Biomechanics 21, 45-54.
Walker, P. S. and Erkman, M. J. (1975) The role of the menisci in force transmission across the knee. Clin. Orthop. Rel. Res. 109, 184-192.

Yao, J. Q. and Seedhom, B. B. (1991) A new technique for measuring contact areas in human joints-the ' $3 \mathrm{~S}$ technique' Proc. Instn Mech. Engrs 205, 69-72. 